associated with a distinct phenotypic spectrum: a cystogenic cilium-dependent pathway, dependent on defective cleavage of ciliary proteins by cytosolic XPNPEP3, and a mitochondriopathy, dependent on defective cleavage of mitochondrial substrates by mitochondrial XPNPEP3. Wow! Future work is needed to define the mitochondrial versus ciliary substrates and roles for hitherto rather obscure, spatially defined metallo-exopeptidases, encoded by a single novel human disease gene, XPNPEP3. Investigators concerned with ciliopathies and mitochondriopathies should stay tuned.

\section{Acknowledgments}

The author is supported by NIH grants 5R01DK056077，5R01DK060043, 5R01DK073960, 3U01DK060995, and 1U01DK085688.

Address correspondence to: Erwin P. Böttinger, Charles R. Bronfman Institute for Personalized Medicine, and Department of Medicine, Mount Sinai School of Medicine, One Gustave L. Levy Pl.,
Box 1003, New York, NY 10029. Phone: 212.241.0800; Fax: 212.849.2643; E-mail: erwin.bottinger@mssm.edu.

1. Pazour GJ, Witman GB. The vertebrate primary cilium is a sensory organelle. Curr Opin Cell Biol. 2003;15(1):105-110.

2. Gerdes JM, Davis EE, Katsanis N. The vertebrate primary cilium in development, homeostasis, and disease. Cell. 2009;137(1):32-45.

3. Singla V, Reiter JF. The primary cilium as the cell's antenna: signaling at a sensory organelle. Science. 2006;313(5787):629-633.

4. Tobin JL, Beales PL. The nonmotile ciliopathies. Genet Med. 2009;11(6):386-402.

5. Scholey JM. Intraflagellar transport motors in cilia: moving along the cell's antenna. J Cell Biol. 2008;180(1):23-29.

6. Rohatgi R, Milenkovic L, Scott MP. Patched1 regulates hedgehog signaling at the primary cilium. Science. 2007;317(5836):372-376.

7. Corbit KC, Aanstad P, Singla V, Norman AR, Stainier DY, Reiter JF. Vertebrate smoothened functions at the primary cilium. Nature. 2005;437(7061):1018-1021.

8. Gerdes JM, Katsanis N. Ciliary function and Wnt signal modulation. Curr Top Dev Biol. 2008;85:175-195.

9. Hildebrandt F, Attanasio M, Otto E. Nephronophthisis: disease mechanisms of a ciliopathy. JAm Soc Nephrol. 2009;20(1):23-35.

10. Lancaster MA, et al. Impaired Wnt-beta-catenin signaling disrupts adult renal homeostasis and leads to cystic kidney ciliopathy. Nat Med.
2009;15(9):1046-1054.

11. Otto EA, et al. Hypomorphic mutations in meckelin (MKS3/TMEM67) cause nephronophthisis with liver fibrosis (NPHP11). J Med Genet. 2009;46(10):663-670

12. Attanasio M, et al. Loss of GLIS2 causes nephronophthisis in humans and mice by increased apoptosis and fibrosis. Nat Genet. 2007; 39(8):1018-1024.

13. O'Toole $\mathrm{J}$, et al. Individuals with mutations in $X P N P E P 3$, which encodes a mitochondrial protein, develop a nephronophthisis-like nephropathy. J Clin Invest. 2010;120(3):791-802.

14. Ersahin C, Szpaderska AM, Orawski AT, Simmons WH. Aminopeptidase P isozyme expression in human tissues and peripheral blood mononuclear cell fractions. Arch Biochem Biophys. 2005;435(2):303-310.

15. Kim KS, Kumar S, Simmons WH, Brown NJ. Inhibition of aminopeptidase $\mathrm{P}$ potentiates wheal response to bradykinin in angiotensin-converting enzyme inhibitor-treated humans. J Pharmacol Exp Ther. 2000;292(1):295-298.

16. Sayer JA, et al. The centrosomal protein nephrocystin- 6 is mutated in Joubert syndrome and activates transcription factor ATF4. Nat Genet. 2006; 38(6):674-681.

17. Li G, et al. A role for Alstrom syndrome protein, alms1, in kidney ciliogenesis and cellular quiescence. PLoS Genet. 2007;3(1):e8.

18. Sullivan-Brown J, et al. Zebrafish mutations affecting cilia motility share similar cystic phenotypes and suggest a mechanism of cyst formation that differs from pkd2 morphants. Dev Biol. 2008;314(2):261-275.

\title{
Resisting arrest: a switch from angiogenesis to vasculogenesis in recurrent malignant gliomas
}

\author{
Jeffrey P. Greenfield,1,2 William S. Cobb,,1,2 and David Lyden ${ }^{1,3}$
}

${ }_{1}^{1}$ Pediatric Brain Tumor Research and the Children's Cancer and Blood Foundation Laboratories, Champalimaud Metastasis Programme,

2Department of Neurosurgery, and ${ }^{3}$ Department of Pediatrics, Weill Cornell Medical College and Memorial Sloan-Kettering Cancer Center, New York, New York.

\begin{abstract}
The cellular and molecular events that initiate and promote malignant glioma development are not completely understood. The treatment modalities designed to promote its demise are all ultimately ineffective, leading to disease progression. In this issue of the JCI, Kioi et al. demonstrate that vasculogenesis and angiogenesis potentially play distinct roles in the etiology of primary and recurrent malignant gliomas, suggesting that patient therapy should perhaps be tailored specifically against the predominant vasculature pathway at a given specific stage of gliomagenesis.
\end{abstract}

Formation of new blood vessels is an essential component of malignant glioma development and progression. As the mechanisms underlying this crucial element of tumor growth are gradually elucidated, it has become increasingly evident that this vascularity arises through multiple mechanisms, depending on the stage of the

Conflict of interest: The authors have declared that no conflict of interest exists.

Citation for this article: J Clin Invest. 2010; 120(3):663-667. doi:10.1172/JCI42345. tumor and possibly the therapies utilized to combat tumor growth. With unregulated cell proliferation and growing tumor size, there is a need for greater oxygen supply to sustain this growth. As mounting cellular metabolism outstrips the oxygen supply made available via the existing vasculature, hypoxia ensues, triggering the process that has been dubbed the "angiogenic switch." While the additional blood supply initially may be obtained simply by co-option of preexisting vessels, the increasing hypoxia eventually necessitates angiogenesis - the sprouting of local vessels via proliferation of existing endothelial cells. Alternatively, vasculogenesis may occur as factors released from tumor cells increase recruitment of circulating endothelial precursor cells or bone marrow-derived hematopoietic cells, also resulting in the formation of new vessels to supply the tumor. Understanding the balance between angiogenesis and vasculogenesis lies at the very core of elucidating how tumors grow, and is crucial to the development of anti-angiogenic therapies. A better comprehension of this process may also allow for the design of new, more effective therapies to target the pathways tumors employ to sustain their growth. In the current issue of the JCI, Kioi et al. (1) suggest that vasculogenesis - but not angiogenesis - is at the center of the revascularization that occurs during glioma recurrence. In clinical terms, their findings suggest that when gliomas recur after irradiation, as they 


\section{Primary occurrence}

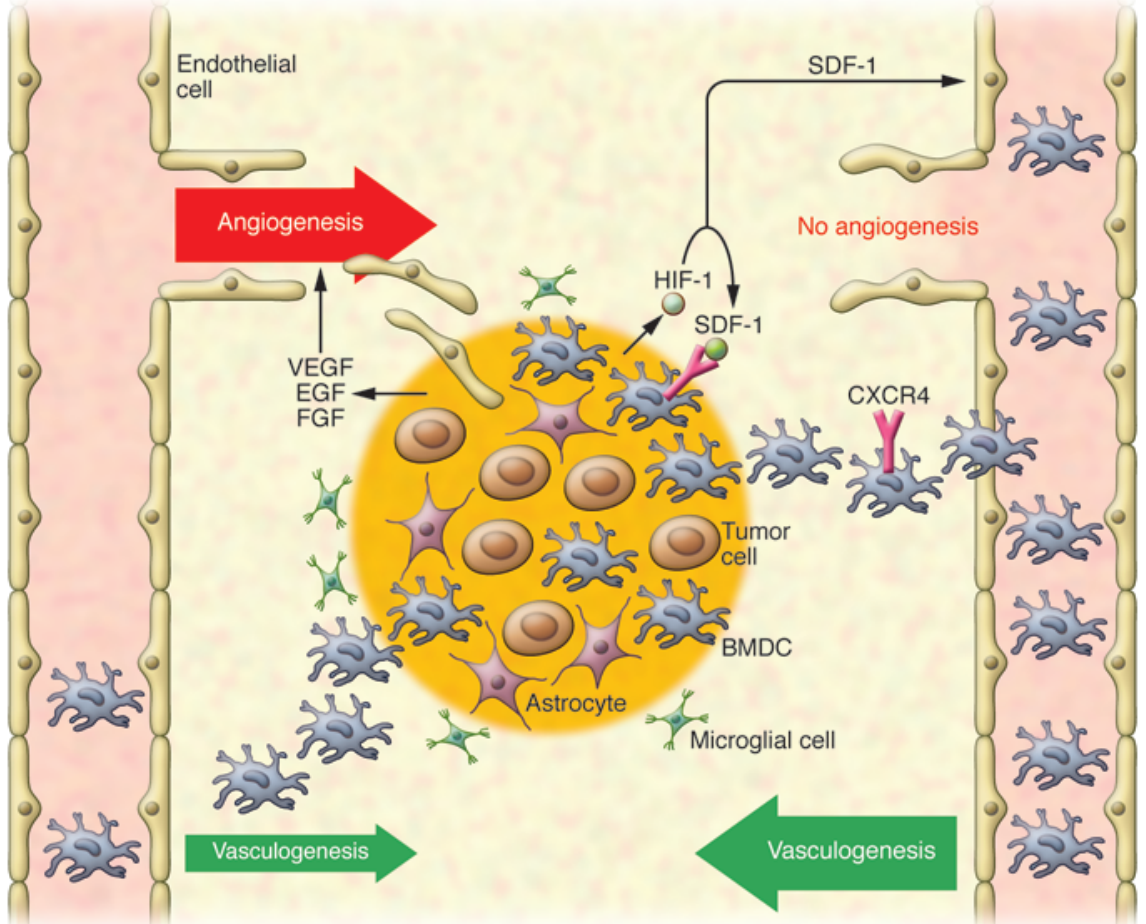

Figure 1

Angiogenesis and vasculogenesis in primary occurrence versus recurrence of malignant glioma. GBM is hypothesized to result from malignant transformation of astrocytes, astrocytic precursors, or neuronal stem cells, and these cells are capable of inducing their own blood supply (25). At initial presentation, it is hypothesized that angiogenesis is largely responsible for neovascularization of GBM. Release of factors such as VEGF, EGF, and FGF from tumor cells stimulates local sprouting of vessels, while recruitment of BMDCs likely plays a minor role. During tumor recurrence after radiation therapy, local sprouting of vessels is largely abrogated, allowing the recruitment of BMDCs to predominate as the main source of neovasculogenesis. In the current issue of the $\mathrm{JCl}$, Kioi et al. (1) identify a potential vasculogenesis pathway whereby hypoxia within the tumor after radiation therapy stimulates HIF-1 release and subsequently elevates SDF-1. Activation of the CXCR4 receptor by SDF-1 promotes mobilization of bone marrow cells, which are then recruited to form new vessels.

invariably do, oncologists may be targeting the irrelevant pathway of re-vascularization by focusing on anti-angiogenic therapies instead of arresting vasculogenesis. In this commentary, we discuss four related areas within the context of this debate: presently available data on blood vessel formation in glioblastoma, differences in the biology of primary and recurrent gliomas, known information about the role of $\mathrm{CD} 11 \mathrm{~b}^{+}$ cells in vasculogenesis, and how current and future therapies take advantage of this growing knowledge base.

\section{Blood vessel formation in glioblastoma multiforme}

To understand the mechanisms of glioblastoma multiforme (GBM) resistance to anti-angiogenesis therapies, one must appreciate how these malignant tumors initially develop their vascular supply. The majority of data suggest angiogenesis, as opposed to vasculogenesis, as the primary etiology of neovascularization in gliomas (2-4). Murine models addressing the role of bone marrow-derived cells (BMDCs) in other cancers have shown minimal, but varied, contributions of non-locally derived cells to the developing vasculature (5). This is in contrast to studies by our group and others that have shown that early vessel formation occurs by the recruitment of BMDCs and that vasculogenesis dominates new vessel formation (6). It is likely that the relative contribution of angiogenic and vasculogenic path- ways is tumor type-specific and reflects differences in species, models, and even antibody selection and techniques utilized to detect BMDCs. Kioi et al. point out that

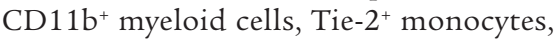
VEGFR $1^{+}$hemangiocytes, and tumor-associated macrophages have all been observed to be incorporated within tumors, particularly in the perivascular regions (1). The unknown factors in the human disease that remain preclude a full understanding of the cues and timing of this recruitment, and of the relative contribution of these different BMDCs.

In the Kioi et al. study (1), the thesis of the article rests on what the authors describe as the paradox of exquisite sensitivity of brain tumor vasculature to irradiation, with resistance to the very therapy used to treat it inevitably emerging. To grasp this idea, one must first appreciate how difficult a GBM is to destroy. The current standard therapeutic regimen for patients with GBM entails a combined approach of maximal surgical resection, temozolomide (an orally administered alkylating chemotherapeutic agent), and simultaneous cranial irradiation. However, most patients experience tumor recurrence, at which point treatment regimens vary dramatically. The combined outcome is a median length of survival of 15 months and a 2-year survival of approximately $25 \%$ (7). Irradiation is unquestionably effective at defeating the most rapidly dividing tumor cells. Nonetheless, its effect on the tumor local microenvironment has received less attention. In the brain, irradiation has been shown to cause gliosis through the disruption of the blood brain barrier and the release of inflammatory factors by microglia $(8,9)$. In breast cancer patients who have received focal irradiation, fibrosis and tissue stiffening have been shown to promote tumor cell growth and invasiveness (10). Thus, it is conceivable that irradiation directed at GBM, while destabilizing existing vessels and destroying tumor cells, could theoretically induce unintended changes in the local microarchitecture, promoting the growth of surviving tumor cells and actually initiating the recruitment of cells that will revascularize the recurrent and ultimately fatal tumor burden. This paradigm of radiation potentiating recurrence could have similar theoretical applicability to metastatic disease in adults and in diseases prone to dissemination, such as medulloblastoma in children. 


\section{Table 1}

The potential targets and related anti-angiogenesis and anti-vasculogenesis therapies in the treatment of glioblastoma and other cancers

\begin{tabular}{|c|c|}
\hline Target & Therapeutic agent \\
\hline \multicolumn{2}{|l|}{ Angiogenesis ${ }^{A}$} \\
\hline VEGF-A & Bevacizumab, aflibercept \\
\hline VEGFR & Cediranib, pazopanib, sorafenib, sunitinib, vatalanib, vandetanib \\
\hline PDGFR & Dasatinib, imatinib, tandutinib \\
\hline EGFR & Cetuximab, erlotinib, gefitinib, nimotuzumab \\
\hline FGFR & Brivanib \\
\hline PIGF & Anti-PIGF Ab \\
\hline HIF-1 & EZN-2968, NSC-134754 \\
\hline HGF-SF/cMet & AMG102 \\
\hline \multicolumn{2}{|l|}{ Vasculogenesis } \\
\hline SDF-1/CXCR4 & AMD3100 \\
\hline Ang-1/Tie-2 & ANG797 \\
\hline Gr-1+ cells & RB6-8C5 \\
\hline CD11b/CD14/CD146+ cells & Anti-CD marker Ab \\
\hline VE-cadherin & Antibody E4B9 \\
\hline CCR6 & Anti-CCR6 Ab \\
\hline $\begin{array}{l}\text { Integrins ( } \alpha 4-\beta 1 / V L A-4 \text {, } \\
\quad \alpha V-\beta 3, \alpha V-\beta 3, \alpha 5-\beta 1)\end{array}$ & Cilengitide, Nischarin, Vitaxin \\
\hline NG2 & Anti-NG2 Ab \\
\hline MMP9 & MMP9 inhibitor I, compound 5a \\
\hline Bv8 & Anti-Bv8 Ab \\
\hline G-CSF & Anti-G-CSF Ab \\
\hline \multicolumn{2}{|c|}{$\begin{array}{l}\text { Many agents have affinity for multiple targets. Ang-1, angiopoietin-1; Bv8, Bombina variegata } \\
\text { peptide 8; FGFR, FGF receptor; Gr-1, granulocyte differentiation antigen } 1 \text {; HGF-SF, hepatocyte } \\
\text { growth factor/scatter factor; NG2, neuronal/glial } 2 \text { protein; PIGF, placental growth factor; Tie-2, tyro- } \\
\text { sine kinase with immunoglobulin and EGF homology domains angiopoietin-2 receptor; VLA-4, very } \\
\text { late antigen-4. AMany factors involved in angiogenesis also play a role in vasculogenesis. }\end{array}$} \\
\hline
\end{tabular}

\section{GBM at recurrence: not the same old story}

Kioi et al. (1) investigated their hypothesis by utilizing a murine GBM xenograft model. In this previously described model (11), radiation causes endothelial cell death and a defined loss of local blood vessels within the tumor bed. The resultant increased hypoxia in the postirradiation state initiates a cascade of events whereby HIF-1 is elevated, increasing the levels of stromal cell-derived factor-1 (SDF-1). Activation of the SDF-1 receptor CXCR4 subsequently promotes the recruitment of bone marrow-derived $\mathrm{CD} 11 \mathrm{~b}^{+}$cells to the tumor site and formation of new blood vessels. The authors theorize that radiation-induced loss of endothelial cells suppresses angiogenesis. The resultant effect is a state of increased hypoxia that activates the HIF-1 pathway, making vasculogenesis the dominant mechanism of neovascularization after radiation therapy (Figure 1). While the current study demonstrates the importance of HIF-1 in GBM revascularization and recurrence after radiation therapy for GBM, there are other factors that must be considered, particularly the role of endothelial cells in this process. Kioi et al. show that although the majority of the $\mathrm{CD} 1^{+}$endothelial cells are destroyed by irradiation, a not insignificant $25 \%$ of the population remains. Previous data from murine models of fibrosarcoma and melanoma suggest that endothelial cells are eminently capable of surviving irradiation, and, in fact, it is this surviving subpopulation that determines clinical response to radiation therapy via angiogenesis (12). In addition, endothelial cells nesting with GBM cells within the tumor microenvironment release factors important for tumor cell proliferation (13). Therefore, it is a distinct possibility that the surviving population of endothelial cells may be a central factor in driving tumor progression in recurrent GBM. The current study also demonstrates that the blockade of the VEGF signaling pathway after irradiation significantly delays tumor regrowth. Although this effect was not as impressive as the effect of inhibiting the HIF-1 pathway, it is likely that VEGF does in fact contribute to revascularization. This raises several questions concerning the role of these remaining endothelial cells after irradiation. Is resistance a result of cells being in different parts of the cell cycle, or is this surviving population intrinsically more resistant to irradiation than those cells that are killed? Are the resistant cells a different cell population altogether? Do the surviving cells already exist within the tumor niche or within different regions of the tumor? Do they arise from the bone marrow? Does this resistant population further recruit circulating cells? While these questions remain unanswered, they certainly represent an important and exciting area of research that warrants further investigation.

\section{Contribution of CD11b+ cells to glioma vascularization}

Depending on one's viewpoint, BMDCs play an important role in tumor vasculature development either initially or at recurrence. These cells can be found within tumors and circulating in the blood of patients with tumors and are mobilized as a result of factors secreted by tumors. What remains to be elucidated are the respective roles of select subpopulations of BMDCs, and how their integration into developing or recurrent tumors is mediated. The host contribution to tumor progression is reflected in the roles of these tumor-infiltrating cells that contribute to the tumor microenvironment and participate in the neoplastic process by fostering proliferation, survival, and metastasis. The myeloid immune suppressor $\mathrm{Gr}^{+} \mathrm{CD} 11 \mathrm{~b}^{+}$cells of myeloid lineage are significantly increased in the bone marrow of animals bearing large tumors and are found in the blood of patients with cancer, including lung (14), head, and neck cancers (15) and brain tumors $(16,17)$. These cells express CD11b, a marker of myeloid cells of macrophage lineage, and Gr-1, a granulocyte marker. Presumably due to their very low expression levels of MHC class II, these cells cannot present antigens and therefore do not induce antitumor responses. What function do $\mathrm{CD} 11 \mathrm{~b}^{+} \mathrm{Gr}-1^{+}$cells serve in patients with cancer? Gene expression profiling of the cells from the bone marrow and tumors derived from refractory and sensitive models suggests that these cells express a distinct set of genes when primed by refractory tumors (18). Specifically, there is enrichment of inflammatory cytokines and downregulation of TGF- $\beta$ superfamily members including Smad4. There is also a 
large reduction in the number of $\mathrm{CD} 11 \mathrm{~b}^{+}$ cells in bone marrow and tumors in mice harboring refractory tumors. In addition, we know that these cells actively contribute to tumor angiogenesis by producing MMP9 and can differentiate into endothelial cells (19). One other subset of monocytes shown to play an important role, at least in tumor angiogenesis, is that of cells expressing Tie-2, an angiopoietin receptor. These cells have been found circulating in human blood and tumors, and experiments targeting them showed impaired tumor angiogenesis (20). Due to the ubiquitous expression of certain cell-surface markers across myeloid cell populations, it is sometimes difficult to determine whether these proangiogenic cells are distinct or overlapping populations. For example, CD $11 b^{+}$cells can be VEGFR $1^{+}$hematopoietic progenitors or Gr-1+ myeloid suppressor cells. In the study by Kioi et al. (1), the major influx of cells in the irradiated tumors consisted of CD $11 b^{+}$ myelomonocytes. However, their data suggest that the majority of these cells had no VEGFR1 or VEGFR2 expression, and a distinct minority were Gr- $1^{+}$. It is surprising that VEGFR1 expression is so low, and one wonders whether flow cytometry as opposed to immunohistochemistry would have been a more sensitive technique to reveal a population of cells not appreciated here. A further complication for the identity of BMDCs involves the back-andforth trafficking of cell surface proteins to other intracellular locations, such as observed with VEGFR1 (21). In addition, direct measurement of this circulating cell population in the peripheral blood would have been a convincing argument in support of their tumor immunostaining, as we and others have previously documented an increase in BMDCs at the time of recurrence in human patients with GBMs (16, 17). It is worth redirecting attention once again to the radiation-resistant population of endothelial progenitor cells in the Kioi et al. study: $25 \%$ of cells staining positive for CD31 survived 15 Gy of irradiation. The authors suggest that "severe vascular damage" and "decreased vessel perfusion" after irradiation could be the stimulus leading to BMDC recruitment. We suggest, based on observations by others (13), that this resistant population of cells should not be dismissed so quickly and that they need better characterization, visualization, and tracking, since they too may play a role in explaining the BMDCs seen in the recurrent tumors. Are these cells circulating dur- ing radiation and being induced by damage, or are they mobilized from the bone marrow? Are they locally dividing or quiescent, and if they are active, are they derived from a preexisting parent population of

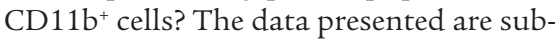
stantial and compelling evidence that the HIF-1/SDF-1 pathway plays an important role in postradiation GBM recurrence, but further characterization of this population of cells is likely to provide substantial insights into this process.

\section{Implications of current and future treatment paradigms}

Dozens of current experimental treatment protocols for recurrent malignant gliomas utilize inhibitors of the VEGF signaling pathway in combination with other therapeutic modalities (Table 1). Bevacizumab in combination with chemotherapy for recurrent glioma produces a radiographic response rate of $66 \%$, and 6 -month progression-free survival (PFS) rates of $40 \%$ are routinely reported (22). These numbers may appear dismal, except when contrasted with the current standard therapy of temozolomide, which has a PFS rate of $21 \%$ (23). Direct inhibition of VEGFR via the oral, nonspecific tyrosine kinase inhibitor cediranib and the specific VEGFR inhibitor sunitinib has been utilized with similar effect. The major benefit of these therapies may not be in their tumoricidal effects, but rather normalization of the tumor vasculature to a degree that allows increased delivery and exposure of the tumor to chemotherapy and a reduction in tumor hypoxia, which might improve efficacy of tumor irradiation.

Resistance to continuing anti-angiogenic therapy may also affect molecules such as SDF-1 and Tie-2; however, it is hard to ignore the central role that the recruitment of $\mathrm{CD} 11 \mathrm{~b}^{+} \mathrm{Gr}-1^{+}$cells to the tumors plays in explaining the underlying refractoriness to anti-VEGF therapy. Some tumors may have an inherent ability to recruit myeloid cells independent of treatment, but in GBM models, anti-VEGF resistance may be due specifically to their ability to recruit myeloid cells. Resistance to antiangiogenic therapy implies that the tumor utilizes alternative pathways and factors discussed above, but also might directly or indirectly involve mobilization of circulating endothelial cells to the tumor. Other therapeutic targets could include less traditional molecules such as Ang-2 (24), because of its destabilizing effect on vasculature through its receptor tyrosine kinase Tie-2, or Delta-like ligand-4, which is important in angiogenesis and signals through the Notch signaling pathway. Chemokines and their receptors are also attractive theoretical targets, and their manipulation might disrupt myeloid cell recruitment via chemotactic signals toward tumor cells. The first generation of antiangiogenic therapies has demonstrated an initial response and limited long-time efficacy against malignant glioma, but what the Kioi et al. (1) and other similar studies suggest is that we need to become more sophisticated in understanding when in the treatment paradigm to use these therapies; how best to monitor their effectiveness; and, most pointedly, how to predict treatment failures before they occur, potentially via simultaneous or staggered blockade of both angiogenic and vasculogenic pathways.

\section{Acknowledgments}

The authors acknowledge grant support from the Children's Cancer and Blood Foundation, the Department of Neurosurgery at Weill Cornell Medical College, the Champalimaud Foundation, The Hartwell Foundation, the Stavros S. Niarchos Foundation, Susan G. Komen for the Cure, the Nancy C. and Daniel P. Paduano Foundation, the National Foundation for Cancer Research, the American Hellenic Educational Progressive Association, the Charles and Meryl Witmer Family Foundation, and the National Cancer Institute.

Address correspondence to: Jeffrey P. Greenfield, Department of Neurosurgery, Weill Cornell Medical College, New York, NY 10021. Phone: 212.746.3941; Fax: 212.746.8423; E-mail: jpgreenf@med. cornell.edu. Or to David Lyden, Department of Pediatrics, Weill Cornell Medical College, New York, NY 10021. Phone: 212.746.3941; Fax: 212.746.8423; E-mail: dc12001@med.cornell.edu.

1. Kioi M, et al. Inhibition of vasculogenesis, but not angiogenesis, prevents the recurrence of glioblastoma after irradiation in mice. J Clin Invest. 2010; 120(3):694-705.

2. Jouanneau E. Angiogenesis and gliomas: current issues and development of surrogate markers. Neurosurgery. 2008;62(1):31-50; discussion 50-52.

3. Tate MC, Aghi MK. Biology of angiogenesis and invasion in glioma. Neurotherapeutics. 2009; 6(3):447-457.

4. Jain RK, et al. Angiogenesis in brain tumours. Nat Rev Neurosci. 2007;8(8):610-622.

5. Gothert JR, et al. Genetically tagging endothelial cells in vivo: bone marrow-derived cells do not contribute to tumor endothelium. Blood. 2004; 
104(6):1769-1777.

6. Lyden D, et al. Impaired recruitment of bone-marrow-derived endothelial and hematopoietic precursor cells blocks tumor angiogenesis and growth. Nat Med. 2001;7(11):1194-1201.

7. Stupp R, et al. Radiotherapy plus concomitant and adjuvant temozolomide for glioblastoma. $N \mathrm{EnglJ}$ Med. 2005;352(10):987-996.

8. Hwang SY, et al. Ionizing radiation induces astrocyte gliosis through microglia activation. Neurobiol Dis. 2006;21(3):457-467.

9. Wilson CM, Gaber MW, Sabek OM, Zawaski JA, Merchant TE. Radiation-induced astrogliosis and blood-brain barrier damage can be abrogated using anti-TNF treatment. Int J Radiat Oncol Biol Phys. 2009;74(3):934-941.

10. Levental KR, et al. Matrix crosslinking forces tumor progression by enhancing integrin signaling. Cell. 2009;139(5):891-906.

11. Ahn GO, Brown JM. Matrix metalloproteinase-9 is required for tumor vasculogenesis but not for angiogenesis: role of bone marrow-derived myelomonocytic cells. Cancer Cell. 2008;13(3):193-205.

12. Garcia-Barros M, et al. Tumor response to radio- therapy regulated by endothelial cell apoptosis. Science. 2003;300(5622):1155-1159.

13. Becher OJ, et al. Gli activity correlates with tumor grade in platelet-derived growth factor-induced gliomas. Cancer Res. 2008;68(7):2241-2249.

14. Almand B, et al. Increased production of immature myeloid cells in cancer patients: a mechanism of immunosuppression in cancer. J Immunol. 2001;166(1):678-689.

15. Young MR, Lathers DM. Myeloid progenitor cells mediate immune suppression in patients with head and neck cancers. Int I Immunopharmacol. 1999;21(4):241-252.

16. Rafat N, Beck G, Schulte J, Tuettenberg J, Vajkoczy P. Circulating endothelial progenitor cells in malignant gliomas. J Neurosurg. 2010;112(1):43-49.

17. Greenfield JP, et al. Surrogate markers predict angiogenic potential and survival in patients with glioblastoma multiforme. Neurosurgery. 2009;64(5):819-826; discussion 826-827.

18. Shojaei F, et al. Tumor refractoriness to anti-VEGF treatment is mediated by CD $11 \mathrm{~b}+\mathrm{Gr} 1+$ myeloid cells. Nat Biotechnol. 2007;25(8):911-920.

19. Yang L, et al. Expansion of myeloid immune sup- pressor $\mathrm{Gr}+\mathrm{CD} 11 \mathrm{~b}+$ cells in tumor-bearing host directly promotes tumor angiogenesis. Cancer Cell. 2004;6(4):409-421.

20. Lewis CE, De Palma M, Naldini L. Tie2-expressing monocytes and tumor angiogenesis: regulation by hypoxia and angiopoietin-2. Cancer Res. 2007;67(18):8429-8432.

21. Zeitlin BD, Ellis LM, Nor JE. Inhibition of vascular endothelial growth factor receptor- $1 / \mathrm{wnt} /$ \{beta\}catenin crosstalk leads to tumor cell death. Clin Cancer Res. 2009;15(24):7453-7455.

22. Norden AD, et al. Bevacizumab for recurrent malignant gliomas: efficacy, toxicity, and patterns of recurrence. Neurology. 2008;70(10):779-787.

23. Yung WK, et al. A phase II study of temozolomide vs. procarbazine in patients with glioblastoma multiforme at first relapse. $\mathrm{Br} J$ Cancer. 2000;83(5):588-593.

24. Norden AD, Drappatz J, Wen PY. Novel anti-angiogenic therapies for malignant gliomas. Lancet Neurol. 2008;7(12):1152-1160.

25. Greenfield JP, et al. Use of human neural tissue for the generation of progenitors. Neurosurgery. 2008;62(1):21-37; discussion 27-30. 